\section{俩 Heighten Science \\ P U B L I C I T I O N S Corporation ISSN \\ 2576-9529}

\title{
Anti-glomerular basement membrane disease: A case report of an uncommon presentation
}

\author{
Natália Silva*, Luís Oliveira, Mónica Frutuoso and Teresa \\ Morgado
}

Nephrology Unit of Hospital Centre of Trás-os-Montes e Alto Douro, Av. Noruega, Lordelo, 5000-508, Vila Real, Portugal

\begin{abstract}
*Address for Correspondence: Natália Silva Hospital Centre of Trás-os-Montes e Alto Douro, Av. Noruega, Lordelo, 5000-508, Vila Real, Portugal, Tel: +351 963563082; Fax: 259300500 Email: nataliasofia_1@hotmail.com

Submitted: 11 March 2019

Approved: 02 April 2019

Published: 03 April 2019

Copyright: () 2019 Silva N, et al. This is an open access article distributed under the Creative Commons Attribution License, which permits unrestricted use, distribution, and reproduction in any medium, provided the original work is properly cited
\end{abstract}

Keywords: Atypical anti-glomerular membrane disease; Crescentic glomerulonephritis; Nephrotic proteinuria

Check for updates

\section{Abstract}

Anti-glomerular basement membrane (anti-GBM) disease is an uncommon autoimmune disease that classically presents as an aggressive necrotizing and crescentic glomerulonephritis (CG), with or without pulmonary hemorrhage, and typically does not relapse. The pathologic hallmark is linear staining of GBM for Immunoglobulin G (IgG) which binds to the alpha-3 chain of type IV collagen. Recent reports have noted the occurrence of anti-GBM disease with atypical clinical presentations. We describe a case of a 22-year-old female presenting an anti-GBM disease with typical histological features with a singular clinical presentation with lower limb edema, elevated serum creatinine and nephrotic range proteinuria. Renal biopsy showed signs of chronicity and direct immunofluorescence microscopy demonstrated diffuse, intense linear positivity for IgG. All serologic tests were negative, including anti-GBM antibodies. Hence, a diagnosis of atypical anti-GBM disease was made. The patient was treated initially with mycophenolate mofetil and corticosteroids. After 3 months she began to aggravate renal function so mycophenolate of mofetil was discontinued and a low dose cyclophosphamide was initiated.

\section{Introduction}

Anti-glomerular basement membrane (GBM) disease is an uncommon autoimmune disease which usually follows a self-limited course [1].

It has been known that the predominant pathogenic autoantibody is the IgG isotype and is directed against to the non-collagenous domain of alpha-3 chain of type IV collagen $[2,3]$. The pathogenic hallmark is the diffuse linear staining of the GBM for IgG on immunofluorescence (IF) microscopy.

These antibodies are detected in $90 \%$ of patients using conventional enzyme-linked immunosorbent assays [4].

Typically, the disease begins abruptly, often with a 'viral-like prodrome', and severe and potentially reversible renal injury, with or without pulmonary hemorrhage [5].

With treatment, it normally subsides, leaving behind a variable degree of lasting glomerular disease, depending on the magnitude of the initial injury and the type of treatment received. Generally, the antibodies anti-GBM disappear from circulation and there is low probability of relapse or recurrence $[5,6]$.

Atypical 'cases' have been published in recent years and the majority describes less severe renal involvement and without detectable antibodies anti-GBM in circulation.

The present case describes an anti-GBM disease with typical pathologic patterns, however, with a very unique presentation. 


\section{Case Report}

A 22-year-old caucasian woman with a history of smoking (2-3 cigarettes a day), excessive consumption of anti- inflammatory drugs and recurrent urinary tract infection during infancy was referred to our hospital with asthenia and edema of the lower limbs. She denied fever, weight loss, hemoptysis, abdominal pain, arthralgia, hematuria or other clinical manifestations. On physical examination, the patient was alert and conscious, well hydrated, and not in repiratory distress. Her vital signs were stable. The remainder of her examination was unremarkable. Laboratory analysis revealed the following values: hemoglobin $8.8 \mathrm{~g} / \mathrm{dL}$, creatinine $2.4 \mathrm{mg} / \mathrm{dL}$, urea $72 \mathrm{mg} / \mathrm{dL}$, albumin $3.1 \mathrm{~g} / \mathrm{dL}$, microhaematuria and proteinuria $4.5 \mathrm{~g} / 24 \mathrm{~h}$. Serum protein electrophoresis showed no monoclonal spike, and her serum free light chain, immunoglobulin and complement levels were normal. Serum electrolytes were also normal. Prothrombin time, partial thromboplastin time, and international normalized ratio showed no alterations. Her serum lipid profile and liver function test were normal.

Serological tests for viral hepatitis B and C, HIV type 1 and 2, antinuclear antibody, anti- ds DNA, and cytoplasmatic and perinuclear anti-neutrophil cytoplasmatic antibodies (c-ANCA and p-ANCA) were all negative. Direct enzyme linked immunoassay for circulating anti-GMB was also negative.

Chest x-ray and renal and abdominal echography showed no alterations. Endoscopy study and computed tomography (TC) scan of the chest and abdomen revealed no abnormalities.

Subsequently, arenalbiopsywas performed.Lightmicroscopy identified 13 glomeruli ( 3 cellular crescents, 6 cellulofibrous crescents and 4 with glomerular sclerosis), with $10 \%$ of interstitial fibrosis and acute tubular necrosis (Figure 1). Fibrinoid necrosis was identified in one of the glomeruli (Figure 2). Immunofluorescence microscopy revealed bright diffuse linear 2+ staining of the glomerular basement membrane with IgG1. There was no detectable staining for IgA, IgM, C1, lambda or kappa light chain. Electron microscopy revealed thickening of the glomerular capillary loops, collapse of the glomerular tuff and focal foot process effacement (Figure 3). There were not seen immune or fibrillar deposits.

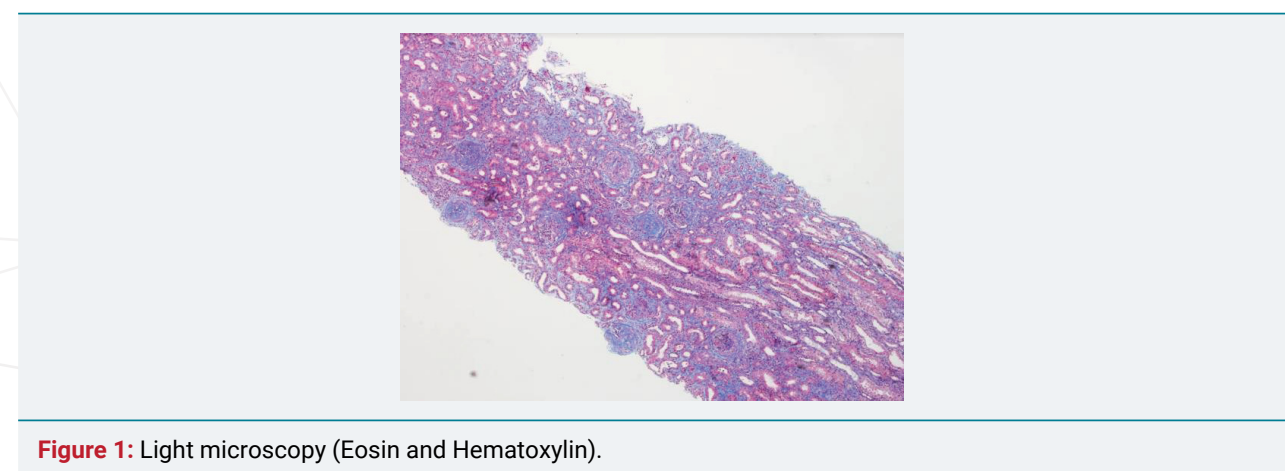

Figure 1: Light microscopy (Eosin and Hematoxylin).

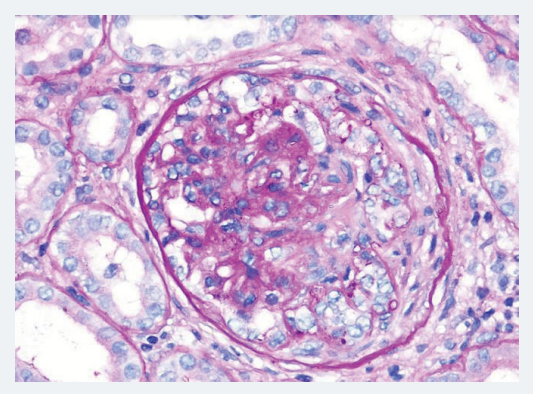

Figure 2: Light microscopy (Periodic Acid Schiff) shows Glomerulus with fibrinoid necrosis. 


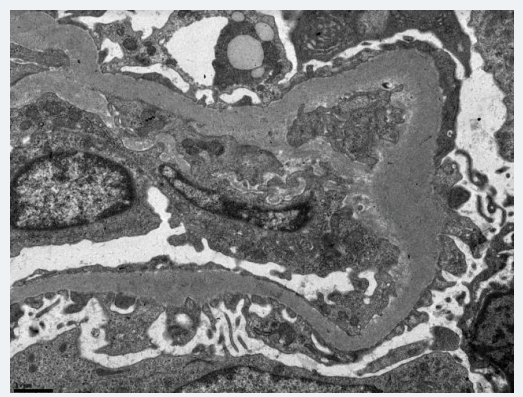

Figure 3: Electron microscopy shows thickening of the glomerular capillary foot process effacement.

Serological tests were tested again with ELISA assay and an additional Western blot test for anti-GBM antibodies, which were both negative.

Based on the aforementioned findings, the diagnosis of atypical anti-GBM disease was made.

Despite being offered treatment with cyclophosphamide, she was treated with $1000 \mathrm{mg} / \mathrm{d}$ i.v. methylprednisolone for 3 days, followed by oral prednisolone $1 \mathrm{mg} /$ $\mathrm{Kg} / \mathrm{d}$ and oral mycophenolate mofetil $500 \mathrm{mg}$ two times a day escalated to a maximum of $750 \mathrm{mg}$ two times a day. The dose of mycophenolate mofetil was not increased due to mild hepatotoxicity. Therapeutic plasma exchange was initiated based on the histologic findings. However, it was discontinued after confirmation of negative antiGBM antibodies (in total the patient had one session of plasmapheresis).

Initially, there was a stabilization of the creatinine values and a decrease of proteinuria. Though, after 3 months she began to aggravate renal function (Creatinine $4,7 \mathrm{mg} / \mathrm{dL})$.

A second kidney biopsy was performed. Light microscopy identified 20 glomeruli, more than $50 \%$ of them with cellulofibrous crescents and 7 with global glomerular sclerosis. There was also 30\% interstitial fibrosis. Immunofluorescence microscopy revealed bright diffuse linear $2+$ staining of the glomerular basement membrane with IgG. There were detected small deposits of C1q and C3.

Mycophenolate of mofetil was discontinued and started a low dose cyclophosphamide $(1,8 \mathrm{mg} / \mathrm{kg} / \mathrm{d})$. At the eleventh day, the patient developed severe neutropenia $(700 / \mu \mathrm{L})$. Cyclophosphamide was suspended immediately. In view of the extensive chronic damage on the biopsy sample, it was decided not to reinitiate additional immunosuppressive therapy. The patient attended a predialysis education program and chose peritoneal dialysis. Tenckhoff catheter was placed and the patient was discharged 2 days later in good clinical condition but with advanced renal disease (Creatinine 6,9 mg/dL; proteinuria $5 \mathrm{~g} / 24 \mathrm{~h}$ ). After 1 month of follow-up she started dialysis (Figure 4).

\section{Discussion}

Anti-GBM antibody disease is a rare organ-specific autoimmune disease, which almost always present as a rapidly progressive glomerulonephritis: acute kidney failure with hypertension, glomerular haematuria and non-nephrotic proteinuria. Pulmonary involvement generally affects approximately 40 to $60 \%$ of this patients and causes symptoms and signs as coughing, shortness of breath, hemoptysis, chest pain and hypoxia. Up to half of affected patients have renal involvement alone and a small minority $(\sim 5 \%)$ has pulmonary limited disease.

The estimated incidence varies between 0.5 and 1.0 cases per million per year [7]. The incidence spikes around 20 and 60-70 years of age [8]. However, younger 


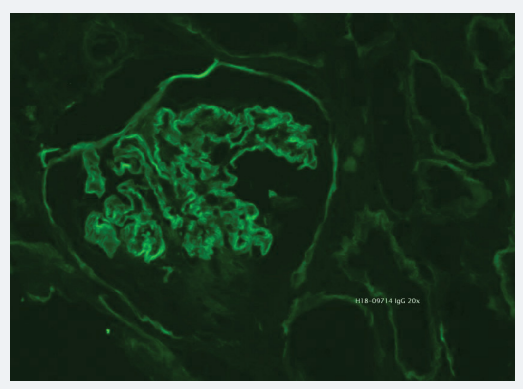

Figure 4: Immunofluorescence microscopy revealed bright diffuse linear staining of the glomerular basement membrane with IgG1.

patients ( $<30$ years) are more likely to present with full constellation of Goodpasture's syndrome, and older patients $(<50$ years) with isolated glomerunephritis $[6,9]$ No ethnic predisposition has been identified [10].

Our patient presented with mild renal failure and nephrotic proteinuria. Serum anti-GMB antibodies were not detected using standard contemporary assays. The two kidney biopsies confirmed the presence of bright diffuse linear 2+ staining of the glomerular basement membrane with IgG1, a histologic finding characteristic of antiGBM antibodies disease. This raises the possibility of antibodies reactive with nonconventional GBM epitopes, of non-typical immunoglobulin subclass, of low affinity or low concentration [11-14].

In this case, before establishing this diagnosis we considered the full differential diagnosis of quasi-linear IgG staining, including fibrillary glomerulonephritis, membranous nephropathy and monoclonal immunoglobulin deposition disease.

According to Nasr et al., atypical anti-GBM has better prognosis than the typical disease. In his cohort of 20 patients with atypical anti-GBM disease, the one year survival rate for renal and patient survival was $85 \%$ and $93 \%$, respectively. Better prognosis can be explained by the lack of pulmonary involvement and low anti-GBM titer, which results in mild renal involvement and less aggressive GN with a smaller number of crescents [15].

It is widely known that the combination therapy of plasmapheresis, corticosteroids and cyclophosphamide has an overall beneficial effect on both patient and renal survival. Though, the standard treatment has shown to be less effective in some of the more aggressive atypical anti-GBM disease cases $[16,17]$.

This case illustrates a rare variant of anti-GBM disease characterized by an indolent presentation, no pulmonary involvement and undetectable anti-GBM antibodies. We emphasize the lack of correlation between clinical and histologic data. Further research is needed to characterize the molecular structure of GBM in these patients and to establish a more universally acceptable therapy for atypical cases.

\section{References}

1. Lerner RA, Glassock RJ, Dixon FJ. The role of anti-glomerular basement membrane antibody in the pathogenesis of human glomerulonephritis. J Exp Med. 1967; 126: 989-1004. Ref.: http://tinyurl.com/y56k3z4p

2. Pedchenko $\mathrm{V}$, Bondar $\mathrm{O}$, Fogo $\mathrm{AB}$, Vanacore $\mathrm{R}$, Voziyan $\mathrm{P}$, et al. Molecular architecture of the Goodpasture autoantigen in anti-GBM nephritis. N Engl J Med. 2010; 363: 343-354. Ref.: http://tinyurl.com/y69wrgpu

3. Kalluri R, Wilson CB, Weber M, Gunwar S, Chonko AM, et al. Identification of the a3 chain of Type IV collagen as the common autoantigen in anti-basement membrane disease and Goodpasture syndrome. J Am Soc Nephrol. 1995; 6: 1178-1185. Ref.: http://tinyurl.com/y3t7k2tn v

4. Troxell ML, Houghton DC. Atypical anti-glomerular basement membrane disease. Clin Kidney J. 2016; 9: 211-221. Ref.: http://tinyurl.com/y3yvgnbs 
5. Cui Z, Turner N, Zhao MH. Antiglomerular basement membrane disease: clinical features and diagnosis. 4th edn. Oxford: Oxford University Press, Oxford Textbook of Clinical Nephrology. 2016; 599-605.

6. Savage CO, Pusey CD, Bowman C, Rees AJ, Lockwood CM. Antiglomerular basement membrane antibody mediated disease in the British Isles 1980-1984. BMJ. 1986; 292: 301-304. Ref.: http://tinyurl.com/y5gvex96

7. Silvariño R, Noboa O, Cervera R. Anti-glomerular basement membrane antibodies. Isr Med Assoc J. 2014; 16: 727-732. Ref.: http://tinyurl.com/y23wsxe2

8. Ohlsson S, Herlitz H, Lundberg S, Selga D, Mölne J, et al. Circulating anti-glomerular basement membrane antibodies with predominance of subclass IgG4 and false negative immunoassay test results in anti-glomerular basement membrane disease. Am J Kidney Dis. 2014; 63: 289-293. Ref.: http://tinyurl.com/y2l6gd6h

9. Levy JB, Turner AN, Rees AJ, Pusey CD. Long-term outcome of anti-glomerular basement membrane antibody disease treated with plasma exchange and immunosuppression. Ann Intern Med. 2001; 134: 1033-1042. Ref.: http://tinyurl.com/y2cq5fvm

10. Taylor DM, Yehia M, Simpson IJ, Thein $\mathrm{H}$, Chang $\mathrm{Y}$, et al. Anti-glomerular basement membrane disease in Auckland. Intern Med J. 2012; 42: 672-676. Ref.: http://tinyurl.com/y2sncagm

11. Cui Z, Zhao MH, Singh AK, Wang HY. Antiglomerular basement membrane disease with normal renal function. Kidney Int. 2007; 72: 1403-1408. Ref.: http://tinyurl.com/y5b664mr

12. Olaru F, Wang XP, Wentian Luo W, Ge L, Miner JH, et al. Proteolysis breaks tolerance toward intact alpha 345(IV) collagen, eliciting novel anti-glomerular basement membrane autoantibodies specific for alpha345NC1 hexamers. J Immunol. 2013; 190: 1424-1432. Ref.: http://tinyurl.com/yywch5a4

13. Yang R, Hellmark T, Zhao J, Cui Z, Segelmark M, et al. Levels of epitope-specific autoantibodies correlate with renal damage in anti-GBM disease. Nephrol Dial Transplant. 2009; 24: 1838-1844. Ref.: http://tinyurl.com/yxwae9oo

14. Segelmark M, Hellmark T, Wieslander J. The prognostic significance in goodpasture's disease of specificity, titre and affinity of anti-glomerular-basement-membrane antibodies. Nephron Clin Pract. 2003; 94: 59-68. Ref.: http://tinyurl.com/yy29j2d3

15. Nasr SH, Collins AB, Alexander MP, Schraith DF, Herrera Hernandez L, et al. The clinicopathologic characteristics and outcome of atypical anti-glomerular basement membrane nephritis. Kidney Int. 2016; 89: 897-908. Ref.: http://tinyurl.com/y342zsyp

16. Yao S, Chen M, Liu Y. Atypical anti-glomerular basement membrane disease with IgA nephropathy: A case report. Int J Clin Exp Med. 2017; 10: 15611-15614. Ref.: http://tinyurl.com/y5ddf9bf

17. Balasubramaniyan T, Dhanapriya J, Dineshkumar T, Maasila AT, Arivazhagan S, Rajasekar $D$, et al Atypical anti-glomerular basement membrane disease superimposed on IgA nephropathy. J Integr Nephrol Androl. 2017; 4: 26-28. Ref.: http://tinyurl.com/y696vryy 